\title{
Pedagogía para la \\ solución de problemas a través de proyectos
}

Raúl Eduardo Gómez Isaza*

\section{Resumen}

En este trabajo se propone un método de enseñanza y aprendizaje para la solución de problemas llamado Método de Proyectos (Project Method). Esto será abordado especificamente desde la visión de Dewey, filósofo y pedagogo norteamericano, que tuvo una gran preocupación por el aprendizaje escolar práctico y su aplicación en las diferentes áreas del comportamiento humano y el desarrollo de las organizaciones. Una teoría no tiene sentido sino cuando puede ser aplicada de manera práctica, al nivel que sea. La teoría guía la práctica, mientras que la práctica corrige la teoría.

Palabras claves: Pedagogía, Escuela Nueva

\section{Abstract:}

This article addresses the Project Method, seen from John Dewey's pedagogical approach.

\section{Introducción}

Las creencias y teorias que están en la base de este método serán tratadas inicialmente, para luego analizar los propósitos y efectos esperados de la utilización del método y de su funcionamiento general. Seguidamente, se verán los roles del profesor y del estudiante, asi como también los principios de reacción de éste método. Después, se abordarán las condiciones que facilitan su funcionamiento. Para terminar, se describirá el método de proyectos aplicado a la Educación en Tecnologia.

\section{Creencias y teorías en la base del método}

Antes de definir el método de proyectos, es importante definir qué es un proyecto. Hay varias interpretaciones del término proyecto; sin embargo se le puede definir de una manera general como una actividad realizada en un medio socio-cultural con un fin especifico.

La noción de proyecto en pedagogía es abordada por Marc Bru y Louis Not (1987) en su libro: ¿Para donde va la pedagogía de proyectos? para desarrollar esta noción, ellos tienen en cuenta los siguientes aspectos: a) el proyecto educativo de 1875 hasta 1975 ; b) la formación y el proyecto de los individuos; c) la escuela y los proyectos de producción. En esta perspectiva, W.H. Kilpatrick clasifica los proyectos en cuatro categorías: a) Proyectos de producción (producers projects) cuyo propósito es producir algún artefacto; b) Proyectos de consumo (consumers projects) cuyo objetivo es el de utilizar algún objeto producido por otros, de aprender a evaluarlo y a apreciarlo; c) Proyectos problemas (problem types) dirigidos a enseñar a solucionar problemas; d) Proyectos de mejoramiento técnico y de aprendizaje (achievement projects) cuyo propósito es, por ejemplo, enseñar a manejar un computador.

Algunos de los aportes de los precursores del método por proyectos,' son los siguientes:

J.J. Rousseau mostró el papel importante que juega el ambiente en el desarrollo del niño. Eso permitió ubicar al alumno en el centro de sus aprendizajes. 
John Dewey, en 1910 probó que utilizando experiencias concretas, el alumno daba respuestas activas y lograba aprendizaje por medio de proyectos para la solución de problemas.

Montessori (1912-1917) supo ubicar el lugar del niño en el centro de su desarrollo, explicando el rol de la maduración espontánea.

Claparede postula una pedagogía pragmática bajo el nombre de "vivencia experimental", valorizando asi el trabajo práctico y manual.

\section{Propósitos y efectos esperados por la utilización del método}

El propósito del método de proyectos en la enseñanza es el de integrar las actividades dentro de la escuela con las que se desarrollan fuera de ella. Es en esta integración que este método tiene significado como una técnica de enseñanza en la escuela. El método de proyectos, propuesto por Dewey en su escuela de aplicación de Chicago, consiste en hacer realizar al alumno un trabajo personal libremente escogido y libremente ejecutado.

Efectos directos: El aprendizaje que precede al comportamiento $y$ al conocimiento adquirido resulta de las transposiciones del mundo real asumidas por los alumnos en su proceso educativo. Los alumnos constatan experimentalmente los efectos, las decisiones y las actitudes tácticas que ellos han adoptado. Los resultados de sus intervenciones les son presentados y los estudiantes son incitados a reflexionar sobre la relación de causa efecto existente en sus decisiones personales. En efecto, diversos tipos de resultados pueden ser adquiridos: saber, saber-hacer; es decir la asimilación de conceptos, el conocimiento de estrategias de solución de problemas, $y$ otros.

Efectos indirectos: El alumno inscrito en el método de proyectos tiene más posibilidades de resistir al olvido, puesto que está confrontando unos objetos significativos. El hecho de que un alumno siga éste proceso o más aún de ser el motor de decisiones conceptuales, de organización, de análisis, de gestión de los trabajos a realizar, permite no solamente desarrollar un espíritu crítico al lograr un gran número de objetivos, sino sobre todo, posibilita el desarrollo de ciertos aspectos tales como: las capacidades (de autonomía, de creatividad), las actitudes (de confianza, de curiosidad, de exploración, entre otros) y las aptitudes (saber - hacer).

Es posible enseñar a todo un grupo permitiendo el desarrollo de la persona; los alumnos adquieren la experiencia y el espíritu de trabajar en grupo, a medida que ellos están en contacto con el proyecto. Además, se presentan otros aprendizajes tales como: la cooperación, la competición, la eficiencia, la toma de decisiones, la facilidad a expresar sus opiniones personales.

\section{Funcionamiento general del método}

Dewey, ubicando la acción antes que el pensamiento, propone el principio fundamental de "Learning by doing", aprender haciendo; de allí los métodos activos. En su pedagogía, este autor propone un método de proyectos en cinco fases:

1) La experiencia de un obstáculo: el profesor debe conducir al alumno a hacerse una o varias ideas acerca de la ecuación a resolver y que describen el proyecto de manera general.

2) El reconocimiento de la ecuación de esquemas intelectuales disponibles: el profesor debe asegurarse de que los alumnos posean el bagaje necesario para desarrollar su proyecto y verificar igualmente su capacidad de solución de problemas.

3) La inspección de datos y de informaciones almacenadas: el alumno debe poseer las facultades que le permitan establecer una dialéctica que se traduzca en acciones prácticas a lo largo del proceso educativo.

4) La elaboración de nuevas vias: el alumno construye él mismo la organización de su propia estructura cognitiva.

5) La prueba de hipótesis: el alumno siendo parte del proyecto, debe ser capaz de buscar nuevas posibilidades hacia las cuales pueda enfocar el proceso.

\section{Rol del profesor y del estudiante y principios de reacción del método.}

El método de proyectos supone que el énfasis es puesto antes que todo, sobre el alumno como responsable de su aprendizaje. Algunos trabajos son sugeridos a los estudiantes, quienes encontrarán alli actividades durante algunas semanas, alrededor de un proyecto. El alumno se esfuerza por crear o fabricar un objeto; debe aprender a servirse de un objeto dado o a poner en práctica una noción. El estudiante se dedica a realizar tareas de solución de algunos problemas o de una dificultad intelectual cualquiera; se esfuerza en perfeccionarse en una cierta técnica que lo habituará a ser un investigador de respuestas que implicará el uso de todas sus fa- 


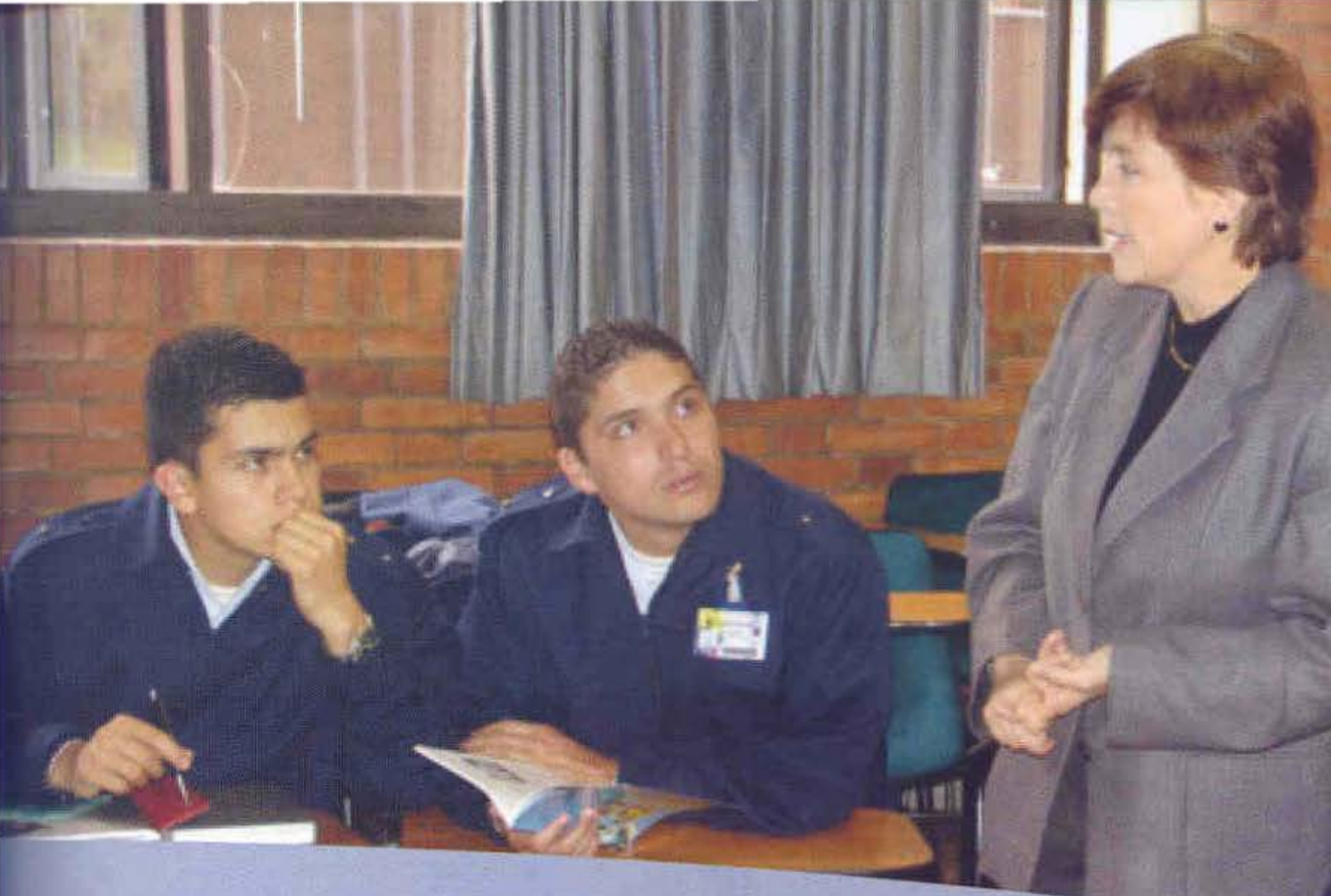


La Educación en Tecnología deberá permitir un enfoque más global, pues el objeto técnico se inserta en un contexto social, industrial y económico. Es por esto que es necesario saber: ¿Dónde se le fabrica? ¿Quién lo fabrica? ¿Cuánto cuesta producirlo? ¿En cuánto se puede vender? ¿Cómo se le repara?.

Un estudio tecnológico tiene dos aspectos: un aspecto intelectual que hace referencia al diseño, su lógica, su creatividad y también a los conocimientos tecnológicos, científicos, matemáticos; y un aspecto práctico, para desarrollar la imaginación, el espíritu de iniciativa, las habilidades, la percepción de formas y el sentido de organización.

A nivel pedagógico, el programa de Educación en Tecnología propone una pedagogía, que a partir de situaciones concretas, requiere la participación activa de los alumnos. En este programa, el método de proyectos llamado "solución de problemas tecnológicos" comprende tres procesos: el proceso de diseño, el proceso de manufactura y el proceso de comercialización de un objeto técnico'. Este trabajo se interesa por el proceso de diseño.

Este proceso que termina con el diseño de un prototipo es privilegiado por los alumnos. Se divide en cinco fases: a) introducción; b) estudio de principios científicos; c) estudio de construcción; d) construcción del prototipo; e) conclusión y evaluación.

En la fase de introducción se realiza tanto la identificación de una necesidad social como el análisis del problema tecnológico. La segunda fase, estudia los principios científicos que constituyen el punto de partida de la construcción del prototipo para responder a la necesidad social identificada precedentemente ${ }^{2}$. Sigue enseguida el estudio de construcción (tercera fase) que incluye todas las especificaciones para fabricar el objeto técnico en el plano práctico. La cuarta fase es la construcción del prototipo propiamente dicho. Una vez que el alumno ha comprendido las fases precedentes, los planos de fabricación y los procedimientos de montaje, podrá pasar a la preparación de las piezas del objeto.

'Québec, Ministére de I'Education. Direction générale du développement pédagogie, Guide pédagogique Initiation a la technologie, 1983, p. 23.

' En las dos primeras fases es prerrequisito hacer esquemas para ilustrar los planos necesarios para la construcción del objeto técnico.
Después de la demostración del profesor sobre el buen uso de herramientas y de técnicas de transformación, vendrá el ensamblaje. El profesor debe insistir igualmente sobre las reglas de seguridad a tener en cuenta. Finalmente, cuando el alumno termina su prototipo, debe verificar con su profesor si éste responde a la necesidad inicial. La fase final, la quinta fase (conclusión y evaluación) permite al profesor y a los alumnos identificar los puntos fuertes y los puntos débiles durante todas las fases del proceso de diseño del prototipo. Ella permitirá al mismo tiempo la orientación del desarrollo de próximos proyectos como la evaluación cualitativa y cuantitativa del trabajo de los alumnos.

\section{Conclusión}

La práctica pedagógica del método por proyectos, inspirándose en todos estos principios orientadores, apoya a los profesores para animar a sus alumnos y ayudarles a tener confianza en sus propios medios. Esta práctica ayudará tanto a profesores como a alumnos a trabajar en conjunto en la solución de todo tipo de problemas. Además, el respeto de estos principios es una necesidad individual para continuar con proyectos futuros. De esta manera, el método de proyectos permitirá al alumno integrar los principios fundamentales de un proyecto durante la construcción de un prototipo cualquiera.

\section{Bibliografía}

BRU, Marc et NOT, Louis, Ou va la pédagogie du projet?, France, Editions Universitaires du Sud, 1987, 306 p.

DEWEY, John, L'école et les méthodes actives", Revue des Sciences de l'Education (Pour lére nouvelle), no 2, avril-juin 1971, pp. 49-57.

MINISTERE DE LEDUCATION DU QUEBEC, GUide pédagogique, Secondaire, Initiation a la technologie, Module 1: Projet d'aménagement architectural, document de travail, Québec, Gouvernement du Québec, Direction générale des programmes, mars 1993,25 p.

MINISTERE DE LEEDUCATION DU QUEBEC, PRogramme d'études. Initiation a la techonologie, Québec, Direction générale du dévelopment pédagogique, 1981, 50 p.

NOT,Louis;"La notion de projet en pédagogie entre 1875 et $1975^{\prime \prime}$, dans Marc Bru et Louis Not, op. cit., P. 1 